\title{
Effective masses for zigzag nanotubes in magnetic fields
}

\author{
Evgeny Korotyaev*
}

August 14, 2018

\begin{abstract}
We consider the Schrödinger operator with a periodic potential on quasi-1D models of zigzag single-wall carbon nanotubes in magnetic field. The spectrum of this operator consists of an absolutely continuous part (intervals separated by gaps) plus an infinite number of eigenvalues with infinite multiplicity. We obtain identities and a priori estimates in terms of effective masses and gap lengths.
\end{abstract}

\section{Introduction and main results}

We consider the Schrödinger operator $\mathscr{H}_{B}=(-i \nabla-\mathscr{A})^{2}+V_{q}$ with a periodic potential $V_{q}$ on the zigzag nanotube $\Gamma^{N} \subset \mathbb{R}^{3}$ (1D models of zigzag single-well carbon nanotubes, see [Ha], [SDD] in a uniform magnetic field $\mathscr{B}=B(0,0,1) \in \mathbb{R}^{3}, B \in \mathbb{R}$. The corresponding vector potential is given by $\mathscr{A}(\mathbf{x})=\frac{1}{2}[\mathscr{B}, \mathbf{x}]=\frac{B}{2}\left(-\mathbf{x}_{\mathbf{2}}, \mathbf{x}_{1}, 0\right), \mathbf{x}=\left(\mathbf{x}_{1}, \mathbf{x}_{2}, \mathbf{x}_{3}\right) \in \mathbb{R}^{3}$. Our model nanotube $\Gamma^{N}$ is a union of edges $\Gamma_{\omega}$ of length 1 , i.e.,

$$
\Gamma^{N}=\cup_{\omega \in \mathcal{Z}} \Gamma_{\omega}, \quad \omega=(n, l, j) \in \mathcal{Z}=\mathbb{Z} \times \mathbb{J} \times \mathbb{Z}_{N}, \quad \mathbb{J}=\{0,1,2\}, \quad \mathbb{Z}_{N}=\mathbb{Z} /(N \mathbb{Z}),
$$

see Fig. 1 and 2, Each edge $\Gamma_{\omega}=\left\{\mathbf{x}=\mathbf{r}_{\omega}+t \mathbf{e}_{\omega}, t \in[0,1]\right\}$ is oriented by the vector $\mathbf{e}_{\omega} \in \mathbb{R}^{3}$ and has starting point $\mathbf{r}_{\omega} \in \mathbb{R}^{\mathbf{3}}$. We have the coordinate $\mathbf{x}=\mathbf{r}_{\omega}+t \mathbf{e}_{\omega}$ and the local coordinate $t \in[0,1]$ (length preserving). We define $\mathbf{r}_{\omega}, \mathbf{e}_{\omega}, \omega=(n, l, j) \in \mathcal{Z}$ by $\mathbf{e}_{n, 0, j}=\mathbf{e}_{0}=(0,0,1)$,

$$
\begin{aligned}
& \mathbf{e}_{n, 1, j}=\varkappa_{n+2 j+1}-\varkappa_{n+2 j}+\frac{\mathbf{e}_{0}}{2}, \quad \mathbf{e}_{n, 2, j}=\varkappa_{n+2 j+2}-\varkappa_{n+2 j+1}-\frac{\mathbf{e}_{0}}{2}, \quad \varkappa_{j}=R_{N}\left(c_{0 j}, s_{0 j}, 0\right), \\
& c_{0 j}=\cos \frac{\pi j}{N}, s_{0 j}=\sin \frac{\pi j}{N}, \quad \mathbf{r}_{n, 0, j}=\varkappa_{n+2 j}+\frac{3 n}{2} \mathbf{e}_{0}, \quad \mathbf{r}_{n, 1, j}=\mathbf{r}_{n, 0, j}+\mathbf{e}_{0}, \quad \mathbf{r}_{n, 2, j}=\mathbf{r}_{n+1,0, j},
\end{aligned}
$$

where $R_{N}=\frac{\sqrt{3}}{4 \sin \frac{\pi}{2 N}}$. The points $\mathbf{r}_{0,0, j}$ are vertices of the regular $\mathrm{N}$-gon $\mathscr{P}_{0}$. The vertical edge $\Gamma_{0,0, j}$ lies on the cylinder $\mathcal{C} \equiv\left\{\mathbf{x} \in \mathbb{R}^{3}: \mathbf{x}_{1}^{2}+\mathbf{x}_{2}^{2}=R_{N}^{2}\right\}$. The starting points $\mathbf{r}_{1,0, j}=\mathbf{r}_{0,2, j}=$ $\varkappa_{1+2 j}+\frac{3}{2} \mathbf{e}_{0}, j \in \mathbb{Z}_{N}$ are the vertices of the regular N-gon $\mathscr{P}_{1}$. $\mathscr{P}_{1}$ arises from $\mathscr{P}_{0}$ by the

*Institut für Mathematik, Humboldt Universität zu Berlin, Rudower Chaussee 25, 12489, Berlin, Germany, e-mail: evgeny@math.hu-berlin.de 


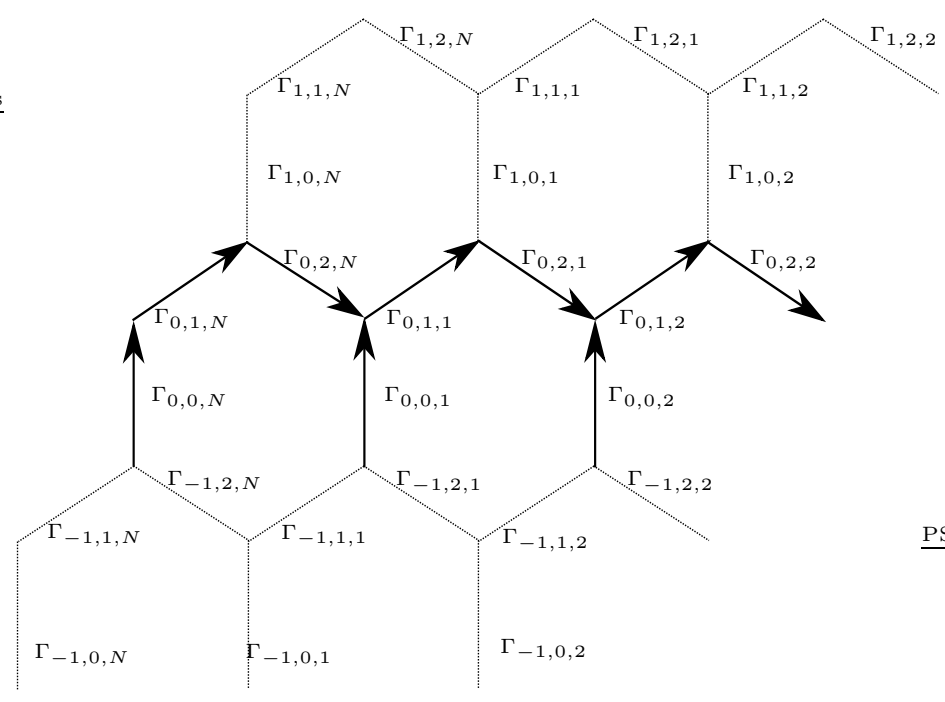

(a)

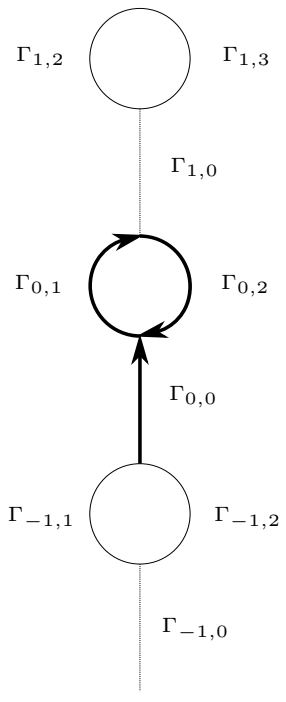

(b)

Figure 1: (a) A piece of a nanotube $\Gamma^{N}$, (b) a nanotube $\Gamma^{1}$. The fundamental domain is marked by a bold line.

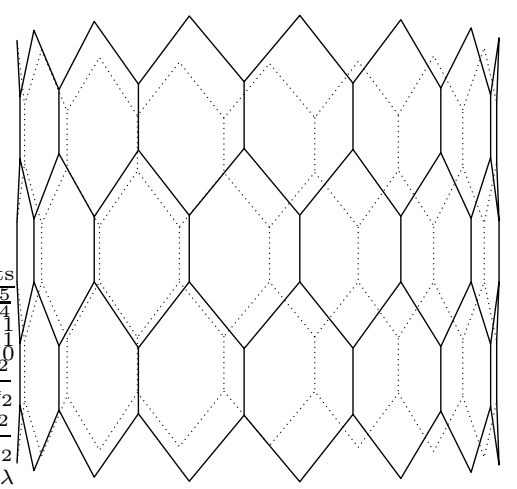

Figure 2: The zigzag nanotube 
following motion: rotate around the axis of the cylinder $\mathcal{C}$ by the angle $\frac{\pi}{N}$ and translate by $\frac{3}{2} \mathbf{e}_{0}$. The non-vertical vectors $\mathbf{e}_{0,1, j}$ and $\mathbf{e}_{0,2, j}$ have positive and negative projections on the vector $\mathbf{e}_{0}$. Repeating this procedure we obtain all edges of $\Gamma^{N}$. Note that each non-vertical edge $\Gamma_{0, l, j}, l=1,2$ (without the endpoints) lies inside the cylinder $\mathcal{C}$. For each function $y$ on $\Gamma^{N}$ we define a function $y_{\omega}=\left.y\right|_{\Gamma_{\omega}}, \omega \in \mathcal{Z}$. We identify each function $y_{\omega}$ on $\Gamma_{\omega}$ with a function on $[0,1]$ by using the local coordinate $t \in[0,1]$.

Our operator $\mathscr{H}_{B}$ on $\Gamma^{N}$ acts in the Hilbert space $L^{2}\left(\Gamma^{N}\right)=\oplus_{\omega} L^{2}\left(\Gamma_{\omega}\right)$ and is given by

$$
\left(\mathscr{H}_{B} f\right)_{\omega}=-\partial_{\omega}^{2} f_{\omega}(t)+q(t) f_{\omega}(t), \quad \partial_{\omega}=\frac{d}{d t}-i a_{\omega}, \quad a_{\omega}(t)=\left(\mathscr{A}\left(\mathbf{r}_{\omega}+t \mathbf{e}_{\omega}\right), \mathbf{e}_{\omega}\right)
$$

see [Ha], SDD], where $\left(V_{q} f\right)_{\omega}=q f_{\omega}, q \in L^{2}(0,1)$ and $\oplus_{\omega} f_{\omega}, \oplus_{\omega} f_{\omega}^{\prime \prime} \in L^{2}\left(\Gamma^{N}\right)$ satisfies The Kirchhoff Magnetic Boundary Conditions: $f$ is continuous on $\Gamma^{N}$ and satisfies

$$
-\partial_{\omega_{3}} f_{\omega_{3}}(1)+\partial_{\omega} f_{\omega}(0)-\partial_{\omega_{4}} f_{\omega_{4}}(1)=0, \quad \partial_{\omega_{1}} f_{\omega_{1}}(0)-\partial_{\omega} f_{\omega}(1)+\partial_{\omega_{2}} f_{\omega_{2}}(0)=0
$$

for all $\omega_{1}=(n+1,0, j), \omega=(n, 1, j), \omega_{2}=(n, 2, j), \omega_{3}=(n, 0, j), \omega_{4}=(n, 2, j-1) \in \mathcal{Z}$. Condition (1.2) means that the sum of derivatives of $f$ at each vertex of $\Gamma^{N}$ equals 0 and the orientation of edges gives the sign \pm .

Such models were introduced by Pauling $[\mathrm{Pa}$ in 1936 to simulate aromatic molecules. They were described in more detail by Ruedenberg and Scherr [RS] in 1953. For physical models see [Ha], [SDD].

For simplicity we will denote $\Gamma_{\omega, 1} \subset \Gamma^{1}$ by $\Gamma_{\omega}$, for $\omega=(n, j) \in \mathcal{Z}_{1}=\mathbb{Z} \times \mathbb{J}$. Thus $\Gamma^{1}=\cup_{\omega \in \mathcal{Z}_{1}} \Gamma_{\omega}$, see Fig 1. The operator $\mathscr{H}_{B}$ is unitarily equivalent to $H(a)=\oplus_{1}^{N} H_{j}(a), a=$ $\frac{3 B}{16} \cot \frac{\pi}{2 N}$ (see [KL1]), where the self-adjoint operator $H_{j}(a)$ acts in the Hilbert space $L^{2}\left(\Gamma^{1}\right)$ and is given by $\left(H_{j}(a) f\right)_{\omega}=-f_{\omega}^{\prime \prime}+q f_{\omega}, f=\left(f_{\omega}\right)_{\omega \in \mathcal{Z}_{1}} \in \mathfrak{D}\left(H_{j}(a)\right)$, where $\mathfrak{D}\left(H_{j}(a)\right)$ consists of all functions $f=\left(f_{\omega}\right)_{\omega \in \mathcal{Z}_{1}},\left(f_{\omega}^{\prime \prime}\right)_{\omega \in \mathcal{Z}_{1}} \in L^{2}\left(\Gamma^{1}\right)$, that satisfy the Kirchhoff conditions

$$
\begin{aligned}
& f_{n, 0}(1)=f_{n, 1}(0)=e^{i a} s^{j} f_{n, 2}(1), \quad f_{n+1,0}(0)=e^{i a} f_{n, 1}(1)=f_{n, 2}(0), \quad s=e^{i \frac{2 \pi}{N}} \\
& -f_{n, 0}^{\prime}(1)+f_{n, 1}^{\prime}(0)-e^{i a} s^{j} f_{n, 2}^{\prime}(1)=0, \quad f_{n+1,0}^{\prime}(0)-e^{i a} f_{n, 1}^{\prime}(1)+f_{n, 2}^{\prime}(0)=0
\end{aligned}
$$

Define the space $\ell^{p}=\left\{h=\left\{h_{n}\right\}_{1}^{\infty}: h_{n} \in \mathbb{C}, \sum_{n \geqslant 1}\left|h_{n}\right|^{p}<\infty\right\}, p \geqslant 1$. Let $\mathfrak{S}_{p}, p \geqslant 1$ be the class of conformal mappings $k: \mathbb{C}_{+} \rightarrow \mathbb{K}(h)=\left\{\lambda \in \mathbb{C}_{+}, \operatorname{Re} \lambda>0\right\} \backslash \cup_{n \geqslant 1}\left[\pi n, \pi n+i h_{n}\right]$, where $h=\left(h_{n}\right)_{1}^{\infty} \in \ell^{p}, h_{n} \geqslant 0$ and $k(\lambda)=i|\lambda|^{\frac{1}{2}}(2+O(1))$ as $\lambda \rightarrow-\infty$. In this case we introduce the sets: spectral bands $\sigma_{n}=\left[\lambda_{n-1}^{+}, \lambda_{n}^{-}\right]=k^{-1}([\pi(n-1), \pi n])$ and gaps $\gamma_{n}=\left(\lambda_{n}^{-}, \lambda_{n}^{+}\right), n \geqslant 1$.

Recall the needed properties of the Hill operator $\widetilde{H} y=-y^{\prime \prime}+q(x) y$ on the real line with a periodic potential $q(x+1)=q(x), x \in \mathbb{R}$, see, e.g., [MO]. We introduce the fundamental solutions $\vartheta(x, \lambda)$ and $\varphi(x, \lambda),(x, \lambda) \in \mathbb{R} \times \mathbb{C}$ of the equation $-y^{\prime \prime}+q(x) y=\lambda y$ satisfying $\vartheta(0, \lambda)=\varphi^{\prime}(0, \lambda)=1, \vartheta^{\prime}(0, \lambda)=\varphi(0, \lambda)=0$. The corresponding Lyapunov function $\Delta$ are given by $\Delta(\lambda)=\frac{\varphi^{\prime}(1, \lambda)+\vartheta(1, \lambda)}{2}, \lambda \in \mathbb{C}$. The spectrum of $\widetilde{H}$ is purely absolutely continuous and consists of intervals $\widetilde{\sigma}_{n}=\left[\widetilde{\lambda}_{n-1}^{+}, \widetilde{\lambda}_{n}^{-}\right], n \geqslant 1$. These intervals are separated by the gaps $\widetilde{\gamma}_{n}=$ $\left(\widetilde{\lambda}_{n}^{-}, \widetilde{\lambda}_{n}^{+}\right)$of length $\left|\widetilde{\gamma}_{n}\right| \geqslant 0$. If a gap $\widetilde{\gamma}_{n}$ is degenerate, i.e. $\left|\widetilde{\gamma}_{n}\right|=0$, then the corresponding 
segments $\widetilde{\sigma}_{n}, \widetilde{\sigma}_{n+1}$ merge. The sequence $\widetilde{\lambda}_{0}^{+}<\widetilde{\lambda}_{1}^{-} \leqslant \widetilde{\lambda}_{1}^{+}<\ldots$ is the spectrum of the equation $-y^{\prime \prime}+q y=\lambda y$ with 2-periodic boundary conditions, that is $y(x+2)=y(x), x \in \mathbb{R}$. Here equality $\widetilde{\lambda}_{n}^{-}=\widetilde{\lambda}_{n}^{+}$means that $\widetilde{\lambda}_{n}^{ \pm}$is an eigenvalue of multiplicity 2 . Note that $\Delta\left(\widetilde{\lambda}_{n}^{ \pm}\right)=$ $(-1)^{n}, n \geqslant 1$. The lowest eigenvalue $\widetilde{\lambda}_{0}^{+}$is simple, $\Delta\left(\widetilde{\lambda}_{0}^{+}\right)=1$, and the corresponding eigenfunction has period 1. The eigenfunctions corresponding to $\widetilde{\lambda}_{n}^{ \pm}$have period 1 if $n$ is even, and they are anti-periodic, that is $y(x+1)=-y(x), x \in \mathbb{R}$, if $n$ is odd. The derivative of the Lyapunov function has a zero $\widetilde{\lambda}_{n}$ in each "closed gap" $\left[\tilde{\lambda}_{n}^{-}, \widetilde{\lambda}_{n}^{+}\right]$, that is $\Delta^{\prime}\left(\widetilde{\lambda}_{n}\right)=0$. Let $\mu_{n}, n \geqslant 1$, be the spectrum of the problem $-y^{\prime \prime}+q y=\lambda y, y(0)=y(1)=0$ (the Dirichlet spectrum). It is well-known that $\mu_{n} \in\left[\widetilde{\lambda}_{n}^{-}, \widetilde{\lambda}_{n}^{+}\right]$. Define the set $\sigma_{D}=\left\{\mu_{n}, n \geqslant 1\right\}$ and note that $\sigma_{D}=\{\lambda \in \mathbb{C}: \varphi(1, \lambda)=0\}$. Define the quasimomentum $\widetilde{k}(\lambda)=\arccos \Delta(\lambda), \lambda \in \mathbb{C}_{+}$. The function $\widetilde{k}(\cdot) \in \mathfrak{S}_{2}$, where the corresponding vector $\left(n^{2} \widetilde{h}_{n}\right)_{1}^{\infty} \in \ell^{2}$ is defined by the equation $\Delta\left(\widetilde{\lambda}_{n}\right)=(-1)^{n} \cosh \widetilde{h}_{n}$. If $\widetilde{\lambda}_{0}^{+}=0$, then $\widetilde{k}$ satisfies (here and below $\sqrt{-1}=i$ )

$$
\begin{gathered}
\widetilde{k}(0)=0, \quad \widetilde{k}(\lambda)=z-\frac{q_{0}+O(1 / z)}{z}, \quad z=\sqrt{\lambda} \in \mathbb{C}_{+}, \quad|z| \rightarrow \infty, \\
\widetilde{k}\left(\mathbb{R}_{-}\right)=i \mathbb{R}_{+}, \widetilde{k}\left(\widetilde{\sigma}_{n}\right)=[\pi(n-1), \pi n], \quad \widetilde{k}\left(\widetilde{\gamma}_{n}\right)=\left[\pi n, \pi n+i \widetilde{h}_{n}\right], \quad \widetilde{k}\left(\lambda_{n}\right)=\pi n+i \widetilde{h}_{n}, n \geqslant 1 .
\end{gathered}
$$

For a self-adjoint operator $H$ we define the set $\sigma_{\infty}(H)=\left\{\lambda: \lambda \in \sigma_{p p}(H)\right.$ is of infinite multiplicity\}. Recall needed results from [KL1]. Let $c_{j}=\cos a_{j}, s_{j}=\sin a_{j}, a_{j}=a+\frac{\pi j}{N}$. If $c_{j} \neq 0$, then the spectrum $\sigma\left(H_{j}(a)\right)=\sigma_{\infty}\left(H_{j}(a)\right) \cup \sigma_{a c}\left(H_{j}(a)\right)$, where $\sigma_{\infty}\left(H_{j}(a)\right)=\sigma_{D}$ and

$$
\sigma_{a c}\left(H_{j}(a)\right)=\left\{\lambda \in \mathbb{R}: \xi_{j}(\lambda, a) \in[-1,1]\right\}=\cup_{n \geqslant 1} \sigma_{j, n}(a), \quad \sigma_{j, n}(a)=\left[\lambda_{j, n-1}^{+}(a), \lambda_{j, n}^{-}(a)\right],
$$

where $\lambda_{j, 0}^{+}<\lambda_{j, 1}^{-} \leqslant \lambda_{j, 1}^{+}<\lambda_{j, 2}^{-} \ldots$ are zeros of the function $\xi_{j}^{2}-1$, and $\xi_{j}$ is the modified Lyapunov functions given by

$$
\xi_{j}=\frac{F+s_{j}^{2}}{c_{j}}, \quad F=\frac{9 \Delta^{2}-\Delta_{-}^{2}-5}{4}, \quad \text { where } \quad \Delta_{-}=\frac{\varphi^{\prime}(1, \cdot)-\vartheta(1, \cdot)}{2}, \quad j \in \mathbb{Z}_{N} .
$$

If $c_{j}=0$, then the spectrum $\sigma\left(H_{j}(a)\right)=\sigma_{\infty}\left(H_{j}(a)\right)=\sigma_{D} \cup\{\lambda \in \mathbb{R}: F(\lambda)=-1\}$.

If $\lambda \in \sigma_{a c}\left(H_{j}(a)\right)$, then the equation $-y^{\prime \prime}+q y=\lambda y$ on $\Gamma^{1}$ with conditions (1.3), (1.4) has a solution $\psi$ such that $\psi_{n+1,0}(0)=e^{i p_{j}(\lambda)} \psi_{n, 0}(0), \psi_{n+1,0}^{\prime}(0)=e^{i p_{j}(\lambda)} \psi_{n, 0}^{\prime}(0), n \in \mathbb{Z}$, where $p_{j}(\lambda)$ is a quasimonentum. The function $\cos p_{j}(\lambda), j \neq 0$ is not entire, and it is define on some Riemann surface. If we take $k_{j}=p_{j}+\frac{\pi j}{N}$, then $\xi_{j}=\cos k_{j}(\lambda)$ is the entire function.

Below we consider only the operator $H_{0}(a), c=\cos a>0$. Due to (1.6) , the results for the operator $H_{0}\left(a+\frac{\pi j}{N}\right)$ give the results for the operator $H_{j}(a)$.

For simplicity we will write $\xi=\xi_{0}$ and $\lambda_{n}^{ \pm}=\lambda_{n}^{ \pm}(a)=\lambda_{0, n}^{ \pm}(a), n \geqslant 0$. The function $\xi^{2}-1$ has only real zeros $\lambda_{n}^{ \pm}$, their labeling is given by $\lambda_{0}^{+}<\lambda_{1}^{-} \leqslant \lambda_{1}^{+}<\lambda_{2}^{-} \leqslant \lambda_{2}^{+}<$.. and if $|c| \notin\left\{\frac{1}{2}, 1\right\}$, then $\lambda_{n}^{-}<\lambda_{n}^{+}$. Here $\sigma_{n}=\left[\lambda_{n-1}^{+}, \lambda_{n}^{-}\right]$are the spectral bands and $\gamma_{n}=\left(\lambda_{n}^{-}, \lambda_{n}^{+}\right)$ 
are the gaps. Moreover, $\lambda_{n}^{ \pm}$satisfy $\xi\left(\lambda_{n}^{ \pm}\right)=(-1)^{n}$ and

$$
\begin{aligned}
& \lambda_{n}^{ \pm}=\lambda_{n}^{0, \pm}+q_{0}+\varepsilon_{n}^{ \pm} \quad \text { as } n \rightarrow \infty, \quad q_{0}=\int_{0}^{1} q(t) d t, \quad n \text { is odd, } \quad c=\frac{1}{2} \\
& \varepsilon_{n}^{ \pm}= \pm\left|\widetilde{q}_{c n}\right|+o\left(n^{-1}\right), \quad \widetilde{q}_{c n}=\int_{0}^{1} q(t) \cos \pi n t d t, \quad \hat{q}_{n}=\int_{0}^{1} q(t) e^{i 2 \pi n t} d t, \quad \hat{q}_{s n}=\operatorname{Im} \hat{q}_{n}, n \text { is even, } \quad c=1, \\
& \varepsilon_{n}^{ \pm}= \pm\left.|| \hat{q}_{n}\right|^{2}-\left.\frac{\hat{q}_{s n}}{9}\right|^{\frac{1}{2}}+\frac{O(1)}{n}, \quad(1 / n) \quad \text { in other cases, }
\end{aligned}
$$

see [KL], KL1]. Here $\lambda_{n}^{0, \pm}$ are 2-periodic eigenvalues for the case $q=0$ given by

$$
\begin{gathered}
\sqrt{\lambda_{0}^{0,+}}=\phi_{0} \in[0, \pi / 2], \quad \sqrt{\lambda_{2 n}^{0, \pm}}=\pi n \pm \phi_{0}, \quad \cos 2 \phi_{0}=\frac{8}{9}\left(c^{2}+c-\frac{7}{8}\right) \in[-1,1], \\
\sqrt{\lambda_{2 n+1}^{0, \pm}}=\pi\left(n+\frac{1}{2}\right) \pm \phi_{1}, \quad \phi_{1} \in[0, \pi / 2], \quad-\cos 2 \phi_{1}=\frac{8}{9}\left(c^{2}-c-\frac{7}{8}\right) \in[-1,1] .
\end{gathered}
$$

The identity $\xi(\lambda)=\cos k(\lambda), \lambda \in \mathbb{C}_{+}$defines an analytic function (the quasimomentum) $k(\lambda), \lambda \in \mathbb{C}_{+}$, see Theorem 1.2. With each edge of the gap $\gamma_{n} \neq \emptyset$, we associate the effective mass $\mu_{0}^{+}, \mu_{n}^{ \pm}$by (we take some branches $k$ such that $k(\lambda) \rightarrow \pi n$ as $\lambda \rightarrow \lambda_{n}^{ \pm}$)

$$
\lambda=\lambda_{n}^{ \pm}+\frac{(k(\lambda)-\pi n)^{2}}{2 \mu_{n}^{ \pm}}(1+o(1)) \quad \text { as } \quad \lambda \rightarrow \lambda_{n}^{ \pm},
$$

and let $\mu_{n}^{ \pm}=0$ if $\left|\gamma_{n}\right|=0$. If $q=0$, then the effective masses $\mu_{n}^{0, \pm}$ are given by (see Sect. 2)

$$
\mu_{0}^{0,+}=\frac{9}{8 c} \frac{\sin 2 \phi_{0}}{\phi_{0}}, \quad \mu_{2 n}^{0, \pm}= \pm \frac{9}{8 c} \frac{\sin 2 \phi_{0}}{\sqrt{\lambda_{2 n}^{0, \pm}}}, \quad \mu_{2 n-1}^{0, \pm}= \pm \frac{9}{8 c} \frac{\sin 2 \phi_{1}}{\sqrt{\lambda_{2 n-1}^{0, \pm}}}, \quad n \geqslant 1,
$$

and $\mu_{n}^{0,+}+\mu_{n}^{0,-}=O\left(1 / n^{2}\right)$ as $n \rightarrow \infty$. Let $F_{0}=\frac{9 \cos 2 \sqrt{\lambda}-1}{8}$. We formulate our first result.

Theorem 1.1. Let $q \in L^{2}(0,1)$. Then the following identities and asymptotics hold true

$$
\begin{gathered}
\mu_{n}^{ \pm}=\mu_{n}^{0, \pm}+\frac{(-1)^{n+1} F_{0}^{\prime \prime}\left(\lambda_{n}^{0, \pm}\right) \varepsilon_{n}^{ \pm}}{c}+\frac{O(1)}{n^{3}} \quad \text { as } \quad n \rightarrow \infty, \\
k^{\prime}(\lambda)^{2}=\frac{1}{2} \sum_{n \geqslant 0, \nu= \pm} \frac{\mu_{n}^{\nu}}{\lambda-\lambda_{n}^{\nu}}, \quad \lambda \neq \lambda_{n}^{ \pm}, n \geqslant 0, \\
\mu_{0}^{+}+\sum_{n \geqslant 1}\left(\mu_{n}^{+}+\mu_{n}^{-}\right)=2, \\
\mu_{2 n}^{ \pm}=2 \sum_{m \geqslant 1, s= \pm}\left(\lambda_{2 m-1}^{s}-\lambda_{2 n}^{ \pm}\right)^{-1}, \quad \mu_{2 n+1}^{ \pm}=2 \sum_{m \geqslant 0, s= \pm}\left(\lambda_{2 m}^{s}-\lambda_{2 n+1}^{ \pm}\right)^{-1}, n \geqslant 0,
\end{gathered}
$$

where the series converges absolutely and uniformly on compact sets in $\mathbb{C} \backslash\left\{\lambda_{n}^{ \pm}, n \geqslant 0\right\}$. 
Remark. Note that $F_{0}^{\prime \prime}\left(\lambda_{n}^{0, \pm}\right) \varepsilon_{n}^{ \pm}=O\left(\varepsilon_{n}^{ \pm} / n^{2}\right)$ as $n \rightarrow \infty$.

Let $\lambda_{n}, n \geqslant 1$ be the zeros of $F^{\prime}$. We have $\xi^{\prime}\left(\lambda_{n}\right)=0$. Recall that $q_{0}=\int_{0}^{1} q(x) d x$.

Theorem 1.2. i) Let $q \in L^{2}(0,1)$ and $\lambda_{0}^{+}=0$. Then a quasimomentum $k(\lambda)=\arccos \xi(\lambda)$, $\lambda \in \mathbb{C}_{+}$belongs to $\mathfrak{S}_{\infty},\left(h_{n}\right)_{1}^{\infty}$ is defined by the equation $\xi\left(\lambda_{n}\right)=(-1)^{n} \cosh h_{n}$, and satisfies

$$
\begin{gathered}
k(0)=0, \quad k(\lambda)=2 z+\log \left(\frac{9}{8 c}\right)^{2}-\frac{q_{0}+O(1 / z)}{z}, \quad z=i|\lambda|^{\frac{1}{2}}, \lambda=z^{2} \rightarrow-\infty, \\
k\left(\mathbb{R}_{-}\right)=i \mathbb{R}_{+}, k\left(\sigma_{n}\right)=[\pi(n-1), \pi n], k\left(\gamma_{n}\right)=\left[\pi n, \pi n+i h_{n}\right], k\left(\lambda_{n}\right)=\pi n+i h_{n}, n \geqslant 1 .
\end{gathered}
$$

ii) Moreover, for each $n \geqslant 1$ the following estimates hold true

$$
\begin{gathered}
h_{n} \leqslant 3 \pi \sqrt{\frac{\left|\gamma_{n}\right|\left|\mu_{n}^{ \pm}\right|}{2}} \leqslant 6 \pi^{2} n\left(\mu_{n}^{+}-\mu_{n}^{-}\right), \\
\left|\gamma_{n}\right| \leqslant(4 \pi n)^{2}\left(\mu_{n}^{+}-\mu_{n}^{-}\right), \\
\left|\gamma_{n}\right| \leqslant 8 \lambda_{n}^{+} \mu_{n}^{+}, \quad\left|\gamma_{n}\right| \leqslant 8 \lambda_{n}^{-}\left|\mu_{n}^{-}\right|+16 \lambda_{n}^{-}\left(\mu_{n}^{-}\right)^{2}, \\
h_{n} \leqslant 4 \pi \sqrt{\lambda_{n}^{ \pm}}\left|\mu_{n}^{ \pm}\right|, \quad h_{n} \leqslant \pi \sqrt{2\left|\gamma_{n}\right|\left|\mu_{n}^{-}\right|}, \quad h_{n} \leqslant 2 \pi \sqrt{\left|\gamma_{n}\right| \mu_{n}^{+}}, \\
h_{n}^{2} \leqslant 2\left|\gamma_{n}\right| \sqrt{\mu_{n}^{+}\left|\mu_{n}^{-}\right|} .
\end{gathered}
$$

If $\gamma_{n}$ is the first non degenerate gap for some $n \geqslant 1$, then $\mu_{0}^{+} \geqslant-\mu_{n}^{-}$.

iii) Moreover, let a spectral interval $\sigma\left(n, n_{1}\right)=\left[\lambda_{n}^{+}, \lambda_{n_{1}}^{-}\right]=\cup_{n+1}^{n_{1}} \sigma_{j}$, where $n_{1}-n$ is a number of the merged components $\sigma_{j}$ which are composed this interval $\sigma\left(n, n_{1}\right)$. Then

$$
\mu_{n}^{+}\left(\lambda_{n_{1}}^{-}-\lambda_{n}^{+}\right) \leqslant 16\left(n_{1}-n\right)^{2}\left(\lambda_{n}^{+}+\lambda_{n_{1}}^{-}\right), \quad\left|\mu_{n_{1}}^{-}\right|\left(\lambda_{n_{1}}^{-}-\lambda_{n}^{+}\right) \leqslant 32\left(n_{1}-n\right)^{2} \lambda_{n_{1}}^{-} .
$$

Remark. 1) There is a big difference beween the quasimomentum for the operator $H_{0}$ and the Hill operator $\widetilde{H}: h \in \ell^{\infty}$ and $\left(n^{2} \widetilde{h}_{n}\right)_{1}^{\infty} \in \ell^{2}$, and, in particular, the unperturbed vector $h^{0} \in \ell^{\infty}$. 2) Note that $n_{1}-n \leqslant 2$ in (1.22).

Below we will sometimes write $\lambda_{n}^{ \pm}(a), \mu_{n}^{ \pm}(a), \xi(\lambda, a), .$. , instead of $\lambda_{n}^{ \pm}, \mu_{n}^{ \pm}, \xi(\lambda), .$. , when several magnetic fields are being dealt with.

Theorem 1.3. Let $q \in L^{2}(0,2)$ and $a \in\left[0, \frac{\pi}{2}\right), n \geqslant 0$. Then

$$
\begin{gathered}
h_{n}^{0}(a) \leqslant h_{n}(a), \quad\left|\mu_{n}^{0, \pm}(a)\right| \leqslant\left|\mu_{n}^{ \pm}(a)\right|, \quad\left|\sigma_{n}^{0}(a)\right| \geqslant\left|\sigma_{n}(a)\right|, \\
h_{n}(a) \leqslant h_{n}\left(a_{1}\right), \quad\left|\mu_{n}^{ \pm}(a)\right| \leqslant\left|\mu_{n}^{ \pm}\left(a_{1}\right)\right|, \quad\left|\sigma_{n}^{0}(a)\right| \geqslant\left|\sigma_{n}\left(a_{1}\right)\right|, \quad \text { all } \quad \frac{\pi}{3} \leqslant a \leqslant a_{1} \leqslant \frac{\pi}{2} .
\end{gathered}
$$

In the present paper we obtain only local estimates, i.e., estimates for fix $n \geqslant 0$. For the Hill operator there exist a priori two sided estimates [K1], [K2]. In order to obtain similar results for the zigzag nanotubes, where $h \in \ell^{\infty}$, we have to study carefully the quasimomentum $k(\cdot)$ as conformal mapping. In our paper we only touch this problem. 


\section{Identities and asymptotics}

Recall that $F=\frac{9 \Delta^{2}-\Delta_{-}^{2}-5}{4}$, where $\Delta_{-}=\frac{\varphi^{\prime}(1, \cdot)-\vartheta(1, \cdot)}{2}$ and $\Delta, F$ satisfy $q_{0}=\int_{0}^{1} q(t) d t$,

$$
\begin{gathered}
\Delta(\lambda)=\cos \sqrt{\lambda}+\frac{q_{0} \sin \sqrt{\lambda}}{2 \sqrt{\lambda}}+\frac{O\left(e^{|\operatorname{Im} \sqrt{\lambda}|}\right)}{|\lambda|}, \quad \Delta_{-}(\lambda)=\frac{o\left(e^{\mid \operatorname{Im} \sqrt{\lambda \mid}}\right)}{|\lambda|^{\frac{1}{2}}}, \\
F(\lambda)=F_{0}(\lambda)+\frac{9 q_{0}}{8} \frac{\sin 2 \sqrt{\lambda}}{\sqrt{\lambda}}+O\left(\frac{e^{2|\operatorname{Im} \sqrt{\lambda}|}}{|\lambda|}\right), \quad F_{0}(\lambda)=\frac{9 \cos 2 \sqrt{\lambda}-1}{8}, \\
F^{\prime}(\lambda)=F_{0}^{\prime}(\lambda)+\frac{9 q_{0}}{8} \frac{\cos 2 \sqrt{\lambda}}{\lambda}+O\left(\frac{e^{2 \mid \operatorname{Im} \sqrt{\lambda \mid}}}{|\lambda|^{\frac{3}{2}}}\right)
\end{gathered}
$$

as $|\lambda| \rightarrow \infty$, uniformly on bounded sets of $q \in L^{2}(0,1)$ (see $[\mathrm{KL}]$ ).

The identity $\mu_{n}^{ \pm}=-(-1)^{n} \xi^{\prime}\left(\lambda_{n}^{ \pm}\right), n \geqslant 0$ from [KK1] together with $\xi=\frac{F+s^{2}}{c}$ yields

$$
\mu_{n}^{ \pm}=-(-1)^{n} \xi^{\prime}\left(\lambda_{n}^{ \pm}\right)=-(-1)^{n} \frac{F^{\prime}\left(\lambda_{n}^{ \pm}\right)}{c}, \quad n \geqslant 0 .
$$

In the unperturbed case $q=0$ the modified Lyapunov function is given by

$$
\xi^{0}=\frac{F_{0}+s^{2}}{c}, \quad F_{0}=\frac{9 \cos 2 z-1}{8} \quad c=\cos a>0, \quad s=\sin a, \quad z=\sqrt{\lambda} .
$$

The function $\xi^{0}\left(z^{2}\right)$ is $\pi$-periodic and on the period $[0, \pi]$ has a maximum at $z \in 0, \pi$ and a minimun at $z=\frac{\pi}{2}$ :

$$
\begin{gathered}
\max _{x \in \mathbb{R}} \xi^{0}\left(x^{2}\right)=\xi(0)=\frac{1+s^{2}}{c}=\frac{2-c^{2}}{c}>1 \text { if } c \neq 1 \quad \text { and } \max _{x \in \mathbb{R}} \xi^{0}\left(x^{2}\right)=1 \text { if } c=1 \\
\min _{x \in \mathbb{R}} \xi^{0}\left(x^{2}\right)=\xi\left(\pi^{2} / 4\right)=-c-\frac{1}{4 c}<-1 \quad \text { if } c \neq \frac{1}{2} \quad \text { and } \quad \xi^{0}(\pi / 2)=-1 \quad \text { if } c=\frac{1}{2} .
\end{gathered}
$$

We have gaps $\gamma_{n}^{0}=\left(\lambda_{n}^{0,-}, \lambda_{n}^{0,+}\right)$, where $\xi^{0}\left(\lambda_{n}^{0, \pm}\right)=(-1)^{n}$. Using $\xi^{0}\left(\lambda_{n}^{0, \pm}\right)=(-1)^{n}$ we have the following equetion for $z_{n}^{0, \pm}=\sqrt{\lambda_{n}^{0, \pm}}>0$ :

$$
9 \cos 2 z-1+8 s^{2}=8 c(-1)^{n}, \quad \cos 2 z=\frac{8}{9}\left(c^{2}+c(-1)^{n}-\frac{7}{8}\right) \in[-1,1] .
$$

Then 2-periodic eigenvalues $\lambda_{n}^{0, \pm}=\left(z_{n}^{0, \pm}\right)^{2}$ have the form (1.8), i.e.,

$$
\begin{gathered}
z_{0}^{0,+}=\phi_{0} \in\left[0, \frac{\pi}{2}\right], \quad z_{2 n}^{0, \pm}=\pi n \pm \phi_{0}, \quad \cos 2 \phi_{0}=\frac{8}{9}\left(c^{2}+c-\frac{7}{8}\right) \in[-1,1] \\
z_{2 n-1}^{0, \pm}=\pi\left(n-\frac{1}{2}\right) \pm \phi_{1}, \quad \phi_{1} \in[0, \pi / 2], \quad \cos 2\left(\frac{\pi}{2}-\phi_{1}\right)=-\cos 2 \phi_{1}=\frac{8}{9}\left(c^{2}-c-\frac{7}{8}\right) \in[-1,1]
\end{gathered}
$$


for $n \geqslant 1$. The function $k^{0}=\arccos \xi^{0}(\lambda)$ is a conformal mapping from the upper half-plane $\mathbb{C}_{+}$onto a quasimomentum domain $\mathbb{K}\left(h^{0}\right)=\mathbb{C} \backslash \cup_{n \geqslant 1}\left[\pi n, \pi n+i h_{n}^{0}\right]$, where $h=\left(h_{n}^{0}\right)_{1}^{\infty}, h_{n} \geqslant 0$ is defined by the equation $\cosh h_{n}^{0}=(-1)^{n} \xi\left(\lambda_{n}\right) \geqslant 1$ and satisfies

$$
\cosh h_{0}^{0}=\frac{1+s^{2}}{c} \geqslant 1, \quad h_{2 n}^{0}=h_{0}^{0}, \quad \text { and } \quad \cosh h_{1}^{0}=\frac{1+4 c^{2}}{4 c} \geqslant 1, \quad h_{2 n+1}^{0}=h_{1}^{0} .
$$

Using (2.4), we deduce that effective masses for $q=0$ are given by

$$
\mu_{n}^{0, \pm}=(-1)^{n+1} \frac{F^{\prime}\left(\lambda_{n}^{0, \pm}\right)}{c}=\frac{9(-1)^{n}}{8 c} \frac{\sin 2 z_{n}^{0, \pm}}{z_{n}^{0, \pm}}, \quad \lambda_{n}^{0, \pm}=\left(z_{n}^{0, \pm}\right)^{2}, \quad n \geqslant 0 .
$$

Thus

$\mu_{0}^{0,+}=\frac{9}{8 c} \frac{\sin 2 \phi_{0}}{\phi_{0}}, \quad \mu_{n}^{0, \pm}= \pm \frac{9}{8 c} \frac{\sin 2 \phi_{0}}{\left(\frac{\pi n}{2} \pm \phi_{0}\right)}, \quad \mu_{n}^{0,+}+\mu_{n}^{0,-}=-\frac{9 \phi_{0} \sin 2 \phi_{0}}{c 4\left[\frac{(\pi n)^{2}}{4}-\phi_{0}^{2}\right]}<0, \quad n$ is even

and

$$
\mu_{n}^{0, \pm}= \pm \frac{9}{8 c} \frac{\sin 2 \phi_{1}}{\left(\frac{\pi n}{2} \pm \phi_{1}\right)}, \quad \mu_{n}^{0,+}+\mu_{n}^{0,-}=-\frac{9 \phi_{1} \sin 2 \phi_{1}}{c 4\left(\frac{(\pi n)^{2}}{4}-\phi_{1}^{2}\right)}<0, \quad n \quad \text { is odd. }
$$

Proof of Theorem 1.1. Using asymptotics (1.7) we obtain

$$
F^{\prime}\left(\lambda_{n}^{ \pm}\right)=F_{0}^{\prime}\left(\lambda_{n}^{ \pm}\right)+O\left(1 / n^{3}\right), \quad F_{0}^{\prime}\left(\lambda_{n}^{ \pm}\right)=F_{0}^{\prime}\left(\lambda_{n}^{0, \pm}\right)+F_{0}^{\prime \prime}\left(\lambda_{n}^{0, \pm}\right) \varepsilon_{n}^{ \pm}+O\left(1 / n^{3}\right)
$$

as $n \rightarrow \infty$. Combine last asymptotics with $\mu_{n}^{ \pm}=-(-1)^{n} \frac{F^{\prime}\left(\lambda_{n}^{ \pm}\right)}{c}$ (see (2.4) $)$ we get (1.11) .

Using the Cauchy theorem about residues for the function ${k^{\prime}}^{2}=\frac{\xi^{2}}{\xi^{2}-1}$, we deduce that

$$
\frac{1}{2 \pi i} \int_{|\rho|=t} \frac{k^{\prime}(\rho)^{2}}{\rho-\lambda} d \rho=k^{\prime}(\lambda)^{2}-\frac{1}{2} \sum_{\left|\lambda_{n}^{\nu}\right|<t, \nu= \pm} \frac{\mu_{n}^{\nu}}{\lambda-\lambda_{n}^{\nu}}, \quad \text { all } t \neq \lambda_{n}^{ \pm}, n \geqslant 0 .
$$

The identity $\xi=\frac{F+s^{2}}{c}$ and $b_{ \pm}= \pm c-s^{2}$ gives

$$
k^{\prime 2}=\frac{\xi^{\prime 2}}{1-\xi^{2}}=\frac{F^{\prime 2}}{c^{2}\left(c^{2}-\left(F+s^{2}\right)^{2}\right.}=\frac{-F^{\prime 2}}{c^{2}\left(F-b_{-}\right)\left(F-b_{+}\right)} .
$$

Using $\cos 2 \phi_{ \pm}=\frac{1+8 b_{ \pm}}{9} \in[-1,1], \quad \phi_{ \pm} \in\left[0, \frac{\pi}{2}\right]$, we obtain

$$
F_{0}(\rho)-b_{ \pm}=\frac{9}{8}\left(\cos 2 z-\cos 2 \phi_{ \pm}\right)=-\frac{9}{4} \sin \left(z-\phi_{ \pm}\right) \sin \left(z+\phi_{ \pm}\right), \quad z=\sqrt{\rho} .
$$

We need the simple estimates (for each $r \in\left(0, \frac{\pi}{2}\right]$ )

$$
2|\sin z| \geqslant e^{|\operatorname{Im} z|}\left(1-e^{-2 r}\right), \quad \text { any } \quad z \in \mathcal{C}_{r}=\{z \in \mathbb{C}:|z-\pi n| \geqslant r, n \in \mathbb{Z}\} .
$$

Using (2.11),(2.12) we obtain for $\zeta_{1}=z-\phi_{ \pm}, \quad \zeta_{2}=z+\phi_{ \pm} \in \mathcal{C}_{r}$ (we take small $r<<1$ )

$$
\left|F_{0}(\rho)-b_{ \pm}\right|=\frac{9}{4}\left|\sin \zeta_{1} \sin \zeta_{2}\right| \geqslant \frac{9}{4} e^{2|\operatorname{Im} z|}\left(1-e^{-2 r}\right)^{2} \quad \text { as } \quad|\rho| \rightarrow \infty .
$$


Substituting $(\underline{2.2}),(\underline{2.3})$ and (2.13) into (2.10) we deduce that

$$
k^{\prime}(\rho)^{2}=\frac{-F^{\prime}(\rho)^{2}}{c^{2}\left(F(\rho)-b_{-}\right)\left(F(\rho)-b_{+}\right)}=\frac{O\left(\rho^{-1}\right) e^{4|\operatorname{Im} \rho|}}{e^{4|\operatorname{Im} \rho|}(1+o(1))}=O\left(\rho^{-1}\right) \quad \text { as } \quad t \rightarrow \infty
$$

for all $\rho \in\{\mu \in \mathbb{C}:|\mu|=t\} \subset \mathcal{C}_{r}$, where $\sqrt{t}-\phi_{ \pm}, \sqrt{t}-\phi_{ \pm} \in \mathcal{C}_{r}$. Thus we obtain $\int_{|\rho|=t} \frac{k^{\prime}(\rho)^{2}}{\rho-\lambda} d \rho=O(1 / n)$ as $t \rightarrow \infty$, which yields (1.12).

In order to show (1.13) we need the following identities

$$
\begin{gathered}
\frac{\mu_{n}^{+}}{\lambda-\lambda_{n}^{+}}+\frac{\mu_{n}^{-}}{\lambda-\lambda_{n}^{-}}=A_{n}+B_{n}, \quad A_{n}=\frac{\left(\mu_{n}^{+}+\mu_{n}^{-}\right)}{2}\left(\frac{1}{\lambda-\lambda_{n}^{+}}+\frac{1}{\lambda-\lambda_{n}^{-}}\right), \\
B_{n}=\frac{\left(\mu_{n}^{+}-\mu_{n}^{-}\right)}{2}\left(\frac{1}{\lambda-\lambda_{n}^{+}}-\frac{1}{\lambda-\lambda_{n}^{-}}\right)=\frac{\left(\mu_{n}^{+}-\mu_{n}^{-}\right)\left(\varepsilon_{n}^{+}-\varepsilon_{n}^{-}\right)}{2\left(\lambda-\lambda_{n}^{+}\right)\left(\lambda-\lambda_{n}^{-}\right)} .
\end{gathered}
$$

Asymptotics (1.11) give $\sum\left|\mu_{n}^{+}+\mu_{n}^{-}\right|<\infty$ and $\sum\left|\left(\mu_{n}^{+}-\mu_{n}^{-}\right)\left(\varepsilon_{n}^{+}-\varepsilon_{n}^{-}\right)\right|<\infty$, which implies

$$
\lambda\left(\frac{\mu_{0}^{+}}{\lambda-\lambda_{0}^{+}}+\sum_{n \geqslant 1} A_{n}\right) \rightarrow \mu_{0}^{+}+\sum_{n \geqslant 1}\left(\mu_{n}^{+}+\mu_{n}^{-}\right), \quad \text { and } \quad \lambda \sum_{n \geqslant 1} B_{n} \rightarrow 0
$$

as $\lambda \rightarrow-\infty$. These asymptotics together with (1.12), (3.3) yield (1.13).

The Hadamard factorization $\xi(\lambda)+1=\prod_{n \geqslant 0, s= \pm}\left(1-\frac{\lambda}{\lambda_{2 n+1}^{s}}\right)$ gives $\frac{\xi^{\prime}(\lambda)}{\xi(\lambda)+1}=\sum_{n \geqslant 0, s= \pm} \frac{1}{\lambda-\lambda_{2 n+1}^{s}}$. Then the identity (2.4) implies

$$
\mu_{2 n}^{ \pm}=-\xi^{\prime}\left(\lambda_{2 n}^{ \pm}\right)=-\frac{2 \xi^{\prime}\left(\lambda_{2 n}^{ \pm}\right)}{\xi\left(\lambda_{2 n}^{ \pm}\right)+1}=2 \sum_{n \geqslant 0, \nu= \pm} \frac{1}{\lambda_{2 n}^{ \pm}-\lambda_{2 n+1}^{\nu}},
$$

which yields (1.14). The proof for $\mu_{2 n+1}^{ \pm}$is similar.

\section{Conformal mappings and estimates}

Proof of Theorem 1.2. i) We need some results from [MO]. Let a function $f$ be entire and $f\left(z^{2}\right), z \in \mathbb{C}$ of exponential type 2 and $f(\lambda)$ be real on the real line and $f(\lambda)=O(1)$ and $f(-\lambda)=C_{f} e^{2|\lambda|^{\frac{1}{2}}+o(1)}$ as $\lambda \rightarrow+\infty$ for some constant $C_{f}>0$. Assume that all zeros of the function $f^{2}-1$ are real and their labeling is given by $\zeta_{0}^{+}<\zeta_{1}^{-} \leqslant \zeta_{1}^{+}<\zeta_{2}^{-} \leqslant \zeta_{2}^{+}<\ldots$. Then there exists a conformal mapping $k: \mathbb{C}_{+} \rightarrow \mathbb{K}(h)$ for some sequence $h=\left(h_{n}\right)_{1}^{\infty} \in \ell^{\infty}$ such that $f(\lambda)=\cos k(\lambda)$ and $k(\lambda)=\sqrt{\lambda}(2+O(1))$ as $\lambda \rightarrow-\infty$ and if $\zeta_{0}^{+}=0$, then $k$ satisfies $k\left(\mathbb{R}_{-}\right)=i \mathbb{R}_{+}, k\left(\left[\zeta_{n-1}^{+}, \zeta_{n}^{-}\right]\right)=[\pi(n-1), \pi n], k\left(\left[\zeta_{n}^{-}, \zeta_{n}^{+}\right]\right)=\left[\pi n, \pi n+i h_{n}\right], k\left(\zeta_{n}\right)=\pi n+i h_{n}$, $n \geqslant 1$, where $\zeta_{1}<\zeta_{2}<\zeta_{3}<.$. are zeros of $f$ and $\zeta_{n} \in\left[\zeta_{n}^{-}, \zeta_{n}^{+}\right]$for all $n \geqslant 1$. 
The function $\xi$ satisfies these conditions, then the statement i) have been proved and we need only to show (1.15). Identities $\Delta(\lambda)=\cos \widetilde{k}(\lambda)$ and asymptotics $\widetilde{k}(\lambda)=z-\frac{q_{0}+o(1)}{2 z}, z=$ $i y=\sqrt{\lambda}, y \rightarrow \infty$, see [MO], and (2.1) give

$$
F(\lambda)=\frac{9}{8}\left(\cos 2 \widetilde{k}(\lambda)+o\left(\lambda^{-1} e^{2 y}\right)\right), \quad \xi(\lambda)=\frac{9}{8 c} \cos 2 \widetilde{k}(\lambda)+o\left(z^{-1} e^{2 y}\right)=\frac{9}{16 c} e^{-2 i \widetilde{k}(\lambda)+o\left(z^{-1}\right)} .
$$

Then $\cos k(\lambda)=\frac{e^{-i k(\lambda)}}{2}\left(1+O\left(e^{-4 y}\right)\right)$ yields (1.15) .

ii) We have proved the existence of the conformal mapping $k: \mathbb{C}_{+} \rightarrow \mathbb{K}(h)$ for some $h \in \ell^{\infty}$. For such conformal mapping the estimates (1.17), (1.18) were proved in [K1]. Moreover, if $\gamma_{n}$ is the first non degenerate gap for some $n \geqslant 1$, then $\mu_{0}^{+} \geqslant-\mu_{n}^{-}[\mathrm{K} 1$ ].

Let $n \geqslant 1$. We need the following estimates from [KK]

$$
\begin{array}{ll}
\frac{\left|g_{n}\right|}{2} \leqslant h_{n} \leqslant \pi \sqrt{2\left|g_{n}\right|\left|m_{n}^{ \pm}\right|} \leqslant 2 \pi\left|m_{n}^{ \pm}\right|, \quad \text { where } & 2 z_{n}^{ \pm} m_{n}^{ \pm}=\mu_{n}^{ \pm}, \quad z_{n}^{ \pm}=\sqrt{\lambda_{n}^{ \pm}}>0 \\
h_{n}^{2} \leqslant 2\left|g_{n}\right| \sqrt{m_{n}^{+}\left|m_{n}^{-}\right|}, \quad\left|g_{n}\right| \leqslant 2\left|m_{n}^{ \pm}\right|, \quad \text { where }\left(z_{n}^{+}+z_{n}^{-}\right)\left|g_{n}\right|=\left|\gamma_{n}\right| .
\end{array}
$$

Consider $\mu_{n}^{+}$. Using the estimate $\left|g_{n}\right| \leqslant 2\left|m_{n}^{+}\right|$we obtain

$$
\left|\gamma_{n}\right| \leqslant 4\left(z_{n}^{+}+z_{n}^{-}\right) z_{n}^{+} \mu_{n}^{+} \leqslant 8 \lambda_{n}^{+} \mu_{n}^{+},
$$

which yields the first estimate in (1.19) . Consider $\mu_{n}^{-}$. Using the estimate $\left|g_{n}\right| \leqslant 2\left|m_{n}^{-}\right|$and identites from (3.1), (3.2) we obtain

$$
\left|\gamma_{n}\right| \leqslant 4\left(z_{n}^{+}+z_{n}^{-}\right) z_{n}^{-} \mu_{n}^{-} \leqslant 8 \lambda_{n}^{-} \mu_{n}^{-}+4\left|g_{n}\right| z_{n}^{-} \mu_{n}^{-}, \quad\left|g_{n}\right| z_{n}^{-} \leqslant 4 \lambda_{n}^{-} \mu_{n}^{-},
$$

which yields (1.19).

The estimate from (3.1) givees $h_{n} \leqslant 2 \pi\left|m_{n}^{ \pm}\right|=4 \pi \sqrt{\lambda_{n}^{ \pm}}\left|\mu_{n}^{ \pm}\right|$, which gives the first estimate in (1.20). Using estimates

$$
\left|g_{n}\right|\left|m_{n}^{-}\right| \leqslant\left|\gamma_{n}\right|\left|\mu_{n}^{-}\right|, \quad\left|g_{n}\right| m_{n}^{+} \leqslant 2\left|\gamma_{n}\right| \mu_{n}^{+}
$$

and (3.1), (3.2), we deduce that

$$
h_{n} \leqslant \pi \sqrt{2\left|\gamma_{n}\right|\left|\mu_{n}^{-}\right|}, \quad h_{n} \leqslant 2 \pi \sqrt{\left|\gamma_{n}\right| \mu_{n}^{+}},
$$

which yields the last two estimates in (1.20). The identities from (3.1), (3.2) give

$$
\left|g_{n}\right|^{2} m_{n}^{+}\left|m_{n}^{-}\right|=4 z_{n}^{+} z_{n}^{-}\left|g_{n}\right|^{2} \mu_{n}^{+}\left|\mu_{n}^{-}\right| \leqslant\left|\gamma_{n}\right|^{2} \mu_{n}^{+}\left|\mu_{n}^{-}\right|
$$

since $4 z_{n}^{+} z_{n}^{-} \leqslant\left(z_{n}^{+}+z_{n}^{-}\right)^{2}$. This implies (1.21).

iii) We need the estimate (see Theorem 2.4 from [KK])

$$
\left|\mu_{ \pm}\right|\left|\sigma\left(n, n_{1}\right)\right| \leqslant 16\left(n_{1}-n\right)^{2} \sqrt{\lambda_{ \pm}}\left(\sqrt{\lambda_{-}}+\sqrt{\lambda_{+}}\right), \quad \mu_{+}=\mu_{n}^{+}, \lambda_{+}=\lambda_{n}^{+}, \mu_{-}=\mu_{n_{1}}^{-}, \lambda_{-}=\lambda_{n_{1}}^{-},
$$

where $n_{1}-n$ is the number of the merged components which are composed the band $\left(\lambda_{+}, \lambda_{-}\right)$. This estimate and $\lambda_{+}<\lambda_{-}$yields (1.22). 
Proof of Theorem 1.3. Using the estimates $\left|F\left(\lambda_{n}\right)\right| \geqslant\left|F_{0}\left(\lambda_{n}^{0}\right)\right| \geqslant 1$ (see Lemma 3.1 from [KL]) we deduce that $h_{n}^{0} \leqslant h_{n}$ for all $n \geqslant 1$. Applying these facts to the qusimomentums $k, k^{0}$ and using (3.4) we get (1.23).

We show (1.24). If $n$ is even, then $F\left(\lambda_{n}\right) \geqslant 1$ and $f_{n}(c)=\cosh h_{n}=\frac{F\left(\lambda_{n}\right)+s^{2}}{c}=$ $-c+\frac{F\left(\lambda_{n}\right)+1}{c}$ and $f_{n}^{\prime}(c)=-1-\frac{F\left(\lambda_{n}\right)+1}{c^{2}}<-1$.

If $n$ is odd, then $F\left(\lambda_{n}\right) \leqslant-\frac{c^{2}}{4}$ (see Lemma 3.1 from [KL]) and $f_{n}(c)=\cosh h_{n}=$ $-\frac{F\left(\lambda_{n}\right)+s^{2}}{c}=c-\frac{F\left(\lambda_{n}\right)+1}{c}$ and $f_{n}^{\prime}(c)=1+\frac{F\left(\lambda_{n}\right)+1}{c^{2}}$. Assume that $c^{2}<\frac{1}{2}$, then $f_{n}^{\prime}(c) \leqslant \frac{c^{2}-\frac{1}{4}}{c^{2}}<0$. Thus the function $h_{n}(a)$ on the interval $\left[\frac{\pi}{3}, \frac{\pi}{2}\right]$ is increasing and $h_{n}(a)<h_{n}\left(a_{1}\right)$ for all $\frac{\pi}{3} \leqslant a<a_{1} \leqslant \frac{\pi}{2}$. Then estimate (3.4) yields (1.24).

Lemma 3.1. The asymptotics (1.15) and following one hold true

$$
k^{\prime}(\lambda)^{2}=\frac{1}{\lambda}+\frac{q_{0}+o(1)}{\lambda^{2}} \quad \text { as } \quad \lambda \rightarrow-\infty .
$$

Proof. Using (2.1) $-(2.3)$, we obtain

$$
k^{\prime}(\lambda)^{2}=-\frac{\xi^{\prime}(\lambda)^{2}}{\xi(\lambda)^{2}-1}=-\frac{\xi^{\prime}(\lambda)^{2}}{\xi(\lambda)^{2}}\left(1+O\left(e^{2 y}\right)\right), \quad \frac{\xi^{\prime}(\lambda)}{\xi(\lambda)}=\frac{F^{\prime}(\lambda)}{F(\lambda)}\left(1+O\left(e^{2 y}\right)\right),
$$

where $y=\sqrt{-\lambda}>0$. Identities $\Delta(\lambda)=\cos \widetilde{k}(\lambda), z=i y=\sqrt{\lambda}$ and asymptotics $\widetilde{k}(\lambda)=$ $z-\frac{q_{0}+o(1)}{2 z}, \quad \tilde{k}^{\prime}(\lambda)=\frac{1}{2 z}\left(1+\frac{q_{0}+o(1)}{2 \lambda}\right)$, (see [MO]) and (2.1) give

$$
\begin{aligned}
& F(\lambda)=\frac{9}{8}\left(\cos 2 \widetilde{k}(\lambda)+o\left(\lambda^{-1} e^{2 y}\right)\right), \quad F^{\prime}(\lambda)=\frac{9}{8}(-\sin 2 \widetilde{k}(\lambda)) 2 \widetilde{k}^{\prime}(\lambda)+o\left(z^{-3} e^{2 y}\right), \\
& \frac{F^{\prime}(\lambda)}{F(\lambda)}=-\frac{\sin \widetilde{k}(\lambda)}{\cos \widetilde{k}(\lambda)}\left(2 \widetilde{k}^{\prime}(\lambda)+o\left(\lambda^{-1}\right)\right)=i\left(2 \widetilde{k}^{\prime}(\lambda)+o\left(z^{-3}\right)=\frac{i}{z}\left(1+\frac{q_{0}+o(1)}{2 \lambda}\right),\right.
\end{aligned}
$$

which yields (3.3).

Lemma 3.2. Let $k_{1}, k_{2} \in \mathfrak{S}_{\infty}$ and let $h_{1, n} \leqslant h_{2, n}$ for all $n \geqslant 1$. Then the corresponding spectral bands $\sigma_{1, n}, \sigma_{2, n}$ and effective masses $\mu_{1, n}^{ \pm}, \mu_{2, n}^{ \pm}$satisfy

$$
\left|\sigma_{1, n}\right| \geqslant\left|\sigma_{2, n}\right|, \quad\left|\mu_{1, n}^{ \pm}\right| \leqslant\left|\mu_{2, n}^{ \pm}\right|, \quad \text { all } \quad n \geqslant 1 .
$$

Proof. Let $r_{j}(\lambda)=k_{j}^{2}(\lambda), \lambda=\zeta+i \eta \in \mathbb{C}_{+}, j=1,2$, where $k_{j}=u_{j}+i v_{j}$. The function $r_{j}$ is a conformal mapping from $\mathbb{C}_{+}$onto $\mathcal{R}_{j}=\left\{r=k^{2}, k \in \mathbb{K}\left(h_{j}\right)\right\}$. Let $\lambda_{j}(r), r \in \mathcal{R}_{j}$ be the inverse mapping $\lambda_{j}=r_{j}^{-1}$. The function $s_{j}=\operatorname{Im} r_{j}=2 u_{j} v_{j}$ is harmonic, nonnegative in $\mathbb{C}_{+}$ and $s_{j} \in C\left(\overline{\mathbb{C}}_{+}\right)$and $r_{j}$ satisfies

$$
r_{j}(\lambda)=\lambda+C_{j}+\frac{1}{\pi} \int_{\lambda_{j, 1}^{-}}^{\infty} s_{j}(t)\left(\frac{1}{t-\lambda}-\frac{t}{1+t^{2}}\right) d t, \quad C_{j}=-\frac{1}{\pi} \int_{\lambda_{j, 1}^{-}}^{\infty} \frac{s_{j}(t) d t}{t\left(1+t^{2}\right)}, \lambda \in \mathbb{C}_{+},
$$

where $\lambda_{j, 1}^{-}>0$ and $\int_{\lambda_{j, 1}^{-}}^{\infty} \frac{s_{j}(t) d t}{\left(1+t^{2}\right)}<\infty$, see [K1]. In the domain $D_{\varepsilon}=\left\{\lambda \in \mathbb{C}_{+}: \varepsilon \leqslant\right.$ $\arg \lambda \leqslant \pi-\varepsilon\}, 0<\varepsilon<\frac{\pi}{2}$ there is an estimate $|t-\lambda| \geqslant|t| \sin \varepsilon$ for all $t>0$. This and 
(3.5) yields $r_{j}(\lambda)=\lambda(+o(1))$ as $\lambda \in D_{\varepsilon},|\lambda| \rightarrow \infty$. But for any $\varkappa$ there exists a constant $\rho=\rho(\varkappa)>0$ such that $\{\lambda:|\lambda|>\rho\} \cap D_{\varkappa} \subset r_{j}\left(D_{\varepsilon}\right), \quad j=1,2$ for some $\varepsilon<\varkappa<\frac{\pi}{2}$. Then $\lambda_{j}(r)=r(1+o(1)), \quad r=t+i s \in D_{\varkappa}$ as $|r| \rightarrow \infty$, and

$$
\frac{\lambda_{1}\left(r_{2}(i \eta)\right)}{i \eta}=\frac{\lambda_{1}\left(r_{2}(i \eta)\right)}{\left(r_{2}(i \eta)\right)} \frac{\left.r_{2}(i \eta)\right)}{i \eta} \rightarrow 1 \text { as } \eta \rightarrow \infty
$$

which yields $f(i \eta)=\eta(1+o(1))$ as $\eta \rightarrow \infty$. Then the Herglotz Theorem yields

$$
f(\lambda)=\operatorname{Im} \lambda_{1}\left(r_{2}(\lambda)\right) \geqslant \operatorname{Im} \lambda_{2}\left(r_{2}(\lambda)\right)=\eta, \quad \lambda=\zeta+i \eta \in \mathbb{C}_{+} .
$$

Then $\operatorname{Im} \lambda_{1} \geqslant \operatorname{Im} \lambda_{2} \geqslant 0$ in the domain $r_{2}\left(\mathbb{C}_{+}\right)$and $\operatorname{Im} \lambda_{1}(r)=\operatorname{Im} \lambda_{2}(r)=0, r \in \mathbb{R}$ give

$$
\lambda_{1}^{\prime}(r)=\frac{\partial}{\partial s} \operatorname{Im} \lambda_{1}(r) \geqslant \frac{\partial}{\partial s} \operatorname{Im} \lambda_{2}(r)=\lambda_{2}^{\prime}(r), \quad r \in \mathbb{R}, r \neq t_{n}=(\pi n)^{2},
$$

(recall that $r=t+i s$ ) which implies

$$
\left|\sigma_{1, n}\right|=\int_{t_{n-1}}^{t_{n}} \lambda_{1}^{\prime}(r) d r \geqslant \int_{t_{n-1}}^{t_{n}} \lambda_{2}^{\prime}(r) d r=\left|\sigma_{2, n}\right|, \quad n \geqslant 1 .
$$

Moreover, we deduce that

$$
\lambda_{j}(r)-\lambda_{j, n}^{+}=\int_{t_{n}}^{r} \lambda_{j}^{\prime}(r) d r=\frac{\left(r-t_{n}\right)^{2}}{(2 \pi n)^{2} 2 \mu_{j, n}^{+}}(1+o(1)) \quad \text { as } r \rightarrow t_{n}+0,
$$

which yelds $\mu_{1, n}^{+} \leqslant \mu_{2, n}^{+}$. The proof for $\mu_{1, n}^{-}$is similar.

\section{References}

[Ha] Harris P. Carbon Nanotubes and Related Structures, Cambridge Univ. Press., Cambridge, 1999.

[KK] Kargaev, P.; Korotyaev, E. Effective masses and conformal mappings. Comm. Math. Phys. 169(1995), 597-625.

[KK1] Kargaev P.; Korotyaev E. The inverse problem for the Hill operator, a direct approach. Invent. Math. 129(1997), 567-593.

[K1] Korotyaev, E. The estimates of periodic potential in terms of effective masses. Commun. Math. Phys., 183(1997), 383-400.

[K2] Korotyaev, E. Estimates of periodic potentials in terms of gap lengths. Comm. Math. Phys. 197 (1998), no. 3, 521-526.

[KL] Korotyaev, E.; Lobanov, I. Schrödinger operators on zigzag nanotubes, will be published in Ann. H. Poincaré. 
[KL1] Korotyaev, E.; Lobanov, I. Magnetic Schrödinger operators on zigzag nanotubes, preprint 2006.

[MO] Marchenko, V.; Ostrovski, I. A characterization of the spectrum of the Hill operator. Math. USSR Sb. 26 (1975), 493-554.

[Pa] Pauling, L. The diamagnetic anisotropy of aromatic molecules, Journal of Chemical Physics, 4(1936), 673-677.

[RS] Ruedenberg, K.; Scherr, C. Free-electron network model for conjugated systems. I. Theory, The Journal of Chemical Physics, 21(1953), 1565-1581.

[SDD] Saito, R.; Dresselhaus, G.; Dresselhaus, M. Physical properties of carbon nanotubes, Imperial College Press, 1998 